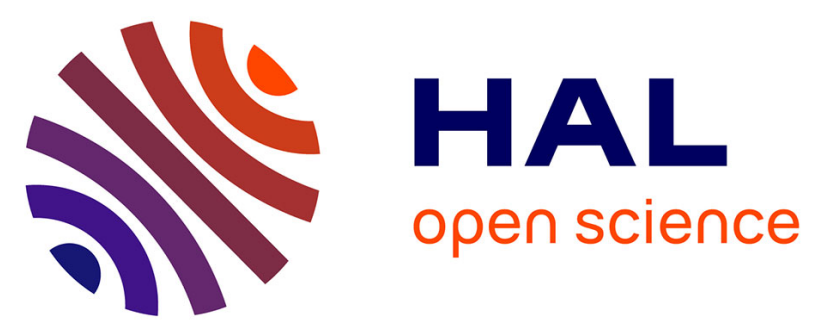

\title{
Sensibilité du pin maritime Pinus pinaster Ait, installé dans des conditions stationnelles différentes, aux attaques de la pyrale du tronc, Dioryctria sylvestrella Ratz (Lepidoptera, Pyralidae). Relations avec quelques caractéristiques de croissance
}

N Carisey, P Ménassieu, P Baradat, B Lemoine, J Levieux

\section{To cite this version:}

N Carisey, P Ménassieu, P Baradat, B Lemoine, J Levieux. Sensibilité du pin maritime Pinus pinaster Ait, installé dans des conditions stationnelles différentes, aux attaques de la pyrale du tronc, Dioryctria sylvestrella Ratz (Lepidoptera, Pyralidae). Relations avec quelques caractéristiques de croissance. Annales des sciences forestières, 1994, 51 (1), pp.67-75. hal-00882933

\section{HAL Id: hal-00882933 https://hal.science/hal-00882933}

Submitted on 1 Jan 1994

HAL is a multi-disciplinary open access archive for the deposit and dissemination of scientific research documents, whether they are published or not. The documents may come from teaching and research institutions in France or abroad, or from public or private research centers.
L'archive ouverte pluridisciplinaire HAL, est destinée au dépôt et à la diffusion de documents scientifiques de niveau recherche, publiés ou non, émanant des établissements d'enseignement et de recherche français ou étrangers, des laboratoires publics ou privés. 


\title{
Sensibilité du pin maritime Pinus pinaster Ait, installé dans des conditions stationnelles différentes, aux attaques de la pyrale du tronc, Dioryctria sylvestrella Ratz (Lepidoptera, Pyralidae). Relations avec quelques caractéristiques de croissance
}

\author{
N Carisey 1, P Ménassieu 1, P Baradat 1, B Lemoine 1, \\ J Levieux 2 \\ ${ }^{1}$ INRA, Pierroton, 33610 Cestas; \\ 2 INRA, Ardon, 45160 Olivet, France
}

(Reçu le 11 mai 1992; accepté le 18 août 1993)

\begin{abstract}
Résumé - Pour évaluer l'impact des caractéristiques de croissance du pin maritime semé dans des conditions stationnelles différentes dans le massif des Landes (France), divers critères reflétant leurs croissances différentielles ont été mesurés dans 15 placettes et corrélés à l'intensité des attaques de Dioryctria sylvestrella. Les arbres localisés dans les placettes les plus productives sont les plus attaqués. Dans une parcelle donnée, les tiges présentant la croissance radiale la plus forte sont préférentiellement attaquées. Par suite, les auteurs suggèrent que la présence d'un liber dénudé et d'un fin rhytidome faciliterait la ponte de la femelle comme la pénétration des larves, la qualité et la quantité du liber facilitant plutôt leur développement.
\end{abstract}

Pinus pinaster / susceptibilité / croissance / Dioryctria sylvestrella / Pyralidae

Summary - Sensitivity of Maritime Pine (Pinus pinaster Ait) to attack by the Pine moth, Dioryctria sy/vestrella Ratz (Lepidoptera, Pyralidae) in a range of site conditions. Relationships to certain growth characteristics. In order to evaluate the differential susceptibility of Pinus pinaster Ait growing in 13-21-yr-old stands where the soil conditions and water content vary gradually with the attacks of the Pine moth Dioryctria sylvestrella (Ratz), several types of measurement were made (basal area, height of the 100 largest trees/ha, etc) and correlated with the intensity of attacks. It appears that the highest productivity stands are the most infected. In a given stand, the trees with the best radial growth are attacked first. It is suggested that the lanval penetration in the inner bark is aided by any naked surface of the tree (linked wounds or cut branches) as well as the presence of a thin bark; the quality and quantity of the inner bark facilitates larval growth. 


\section{INTRODUCTION}

Le massif landais, semé en pin maritime sur plus d'un million d'hectares, constitue la première région productrice de bois résineux en France. Les améliorations dans les techniques d'installation et de gestion sylvicole des peuplements ont permis d'élever en 20 ans la productivité de 4 à 9 $\mathrm{m}^{3} / \mathrm{ha} / \mathrm{an}$ (Inventaire forestier nationa!, 1971 et 1992). L'incidence de l'amélioration génétique dans l'accroissement de la production et de la qualité (rectitude du fût) commencera à être notable à partir de la fin des années 1990 (Baradat et Pastuz$\mathrm{ka}, 1990)$. Cette intensification de la production n'est peut-être pas sans augmenter les risques phytosanitaires, en particulier ceux dus aux attaques de la pyrale du tronc, Dioryctria sylvestrella Ratz, dont la chenille vit aux dépens du liber (Ménassieu et al, 1989).

Dans un tel contexte, le but de ce travail consiste à évaluer l'influence des caractéristiques de croissance de divers peuplements considérés comme représentatifs des milieux écologiques landais sur leur niveau d'infestation. Pour ce faire, nous avons étudié, entre 1985 et 1989 , dans la région de Pissos (Landes), 3000 arbres répartis sur 15 placettes semipermanentes échantillonnées en fonction du degré d'alimentation en eau (lande sèche à lande humide) de leurs sols par le laboratoire de croissance et production (INRA, Bordeaux Cestas).

\section{MATÉRIEL ET MÉTHODES}

\section{Milieux et peuplements}

\section{Localisation géographique}

Une surface de 5000 hectares, intégrée au bassin versant de la rivière la Leyre (région de Pissos, Landes), avait été délimitée afin d'y étudier la répartition spatiale des grandes associations végétales en relation avec les caractéristiques hydrologiques et pédologiques du milieu. Quatre zones représentatives des divers paysages végétaux rencontrés ont été retenues (SaintDidier, 1976).

\section{Caractéristiques écologiques des stations et des peuplements}

Les 4 types de lande sont définis chacun par la présence de 2 ou 3 espèces végétales (tableau I).

La lande humide (LH) abrite les 6 parcelles $121,122,125,127,130$ et 131 ; la mésophile humide (LMH), la parcelle 128; la mésophile sèche (LMS), les 3 parcelles 103,111 et 115 ; et la lande sèche (LS), les 5 parcelles 102, 105, 107, 112 et 113. La surface unitaire des pla-

Tableau I. Principales espèces végétales caractérisant les 4 types de landes étudiées.

\begin{tabular}{lll} 
Type de lande & Espèces végétales & Nom commun \\
\hline Lande humide & Molinia coerulea & Molinie \\
Lanca tetralix & Bruyère à 4 angles \\
Lande mésophile humide & Molinia coerula & Molinie \\
Lande sèche & Pteris aquilina & Fougère aigle \\
& Helianthemum alyssoides & Hélianthème \\
& Calluna vulgaris & Callune
\end{tabular}


cettes de mesures installées dans ces peuplements varie de 8,79 à 19,76 ares et le nombre de tiges en 1988 de 146 à 260 . Ces peuplements, obtenus par semis artificiels, étaient âgés de 12 à 20 ans en 1988 .

\section{Méthodes d'appréciation des attaques, mesures dendrométriques et interprétation des résultats}

\section{Notation d'attaques}

Les notations d'attaques ont toujours été effectuées au printemps après la reprise d'activité des chenilles, la couleur blanc rosé de la coulée de résine fraîche facilitant l'observation. Les critères suivants ont été pris en compte.

\section{Critères quantitatifs}

En 1985, dénombrement des attaques de l'année et de la totalité des attaques antérieures, repérables par un amas caractéristique de résine cristallisée.

De 1986 à 1989, dénombrement annuel des attaques nouvelles.

\section{Critères qualitatifs}

Position des attaques sur l'arbre (verticille, tronc, branche, fourche, crosse et gélivure suite à l'hiver glacial de 1985). Âge des pousses attaquées.

Étant donné le nombre généralement faible d'attaques par placette et par année, nous avons décidé, en vue des traitements statistiques, de cumuler les attaques sur une période minimale de 3 ans. Nous avons ainsi calculé le nombre moyen d'attaques par arbre $T_{a}$ (Suéron, 1982) pour chaque placette ou rapport du nombre d'attaques observées pendant une période donnée $n_{\mathrm{t}}$ au nombre total de tiges de la placette $n_{p}$; le nombre d'attaques par arbre attaqué $T_{b}$ durant une période donnée.

\section{Caractéristiques dendrométriques des peuplements}

Les paramètres suivants ont été mesurés en 1980, puis chaque année de 1984 à 1988 :
- circonférence $c$ de chaque arbre à 1,30 m ;

- hauteur moyenne des arbres dominants $h_{\text {dom }}$ sur la base des 100 gros à l'hectare, cet effectif étant proportionnel à la surface de la placette ;

- nombre de tiges $n_{p}$ par placette ;

- densité de peuplement $N$ (nombre de tiges par hectare).

De tels paramètres, recueillis également sur des peuplements plus âgés, ont servi à l'élaboration d'un modèle de croissance et de production de peuplements en fonction des conditions du milieu et du type de sylviculture (Lemoine, 1991). À l'aide de cette méthode, nous avons déterminé des caractéristiques de peuplement prenant en compte l'influence des pratiques sylvicoles successives, la fertilité du sol et des arbres, soit :

- un indice de vigueur $\mathrm{H}_{16}$ (ou indice de productivité) : hauteur dominante en mètres à 16 ans (moyenne d'âge des 15 peuplements), calculé d'après le modèle; et sur 2 périodes (19801985, 1980-1988) :

- l'accroissement en hauteur dominante $\Delta h_{\text {dom }}$ du peuplement ;

- l'accroissement en surface terrière de l'arbre $\Delta \mathrm{g}$;

- l'accroissement moyen en surface terrière $\overline{\Delta g}$ en $\mathrm{cm}^{2}$ du peuplement;

- la densité de peuplement $N_{\text {moy }}$;

- l'indice de concurrence $L_{\text {moy }}$ : c'est la fonction de concurrence du modèle de croissance faisant jouer la densité $N$ du peuplement et la grosseur de l'arbre moyen dominant (l'équivalent d'un facteur d'espacement). Si $L_{\text {moy }}=1$, on est en croissance libre; si $L_{\text {moy }}=0,80$, la réduction de croissance est de $20 \%$.

\section{Traitements statistiques}

En raison des éclaircies et de la mortalité naturelle, la densité des peuplements a diminué entre 1980 et 1988 . Afin de pouvoir travailler sur un échantillon constant, seule la population des arbres encore présents en 1988 a été prise en compte, en supposant celle-ci représentative du peuplement.

Le traitement des données a été réalisé à l'aide du logiciel statistique Opep (Baradat, 1989). Deux méthodes statistiques ont été utilisées. 
L'analyse factorielle discriminante (AFD) portant sur la totalité des arbres, puis sur l'ensemble des arbres attaqués entre 1980 et 1988 a été utilisée dans le but de comparer les peuplements entre eux sur 2 critères : I'accroissement individuel en surface terrière $\Delta \mathrm{g}$ et le nombre moyen d'attaques par arbre $T_{a}$. Étant donné les caractéristiques particulières d'attaque de cet insecte (pas de phénomène de propagation et faible densité), l'hypothèse d'indépendance entre les individus peut être faite. Cela est une condition nécessaire pour que les nombres moyens d'attaques par arbre puissent être reliés aux autres caractéristiques de chaque placette.

La régression multiple descendante a été employée afin de déterminer le pouvoir prédicteur des différentes caractéristiques de peuplement sur le taux d'attaque.

\section{RÉSULTATS}

\section{Taux d'attaque en fonction de leur emplacement sur l'arbre}

Le tableau II présente en pourcentage les attaques totales de 1986 à 1988, en fonction de leur localisation sur l'arbre. On remarquera la forte proportion d'attaques sur le tronc au niveau du verticille (63\%). L'écorce à cet endroit est plus fissurée que celle du tronc. Elle peut donc faciliter la ponte de la pyrale et l'installation de sa larve.

Tableau II. Pourcentages des attaques de Dioryctria sy/vestrella selon leur localisation sur l'arbre.

Position Attaques (\%)

Verticille

63

Entre-nœud

29

Gélivure

4

Fourche et Crosse

3

Branche
Un examen plus détaillé de la répartition spatiale des attaques en fonction de l'âge de la zone attaquée indique que, dans $84 \%$ des cas, les coulées de résine sont localisées sur la partie supérieure de la tige âgée de 2 à 6 ans. Le maximum d'attaques se trouve sur la zone âgée de 3 à 4 ans (fig 1). Dans $12 \%$ des cas, les attaques ont lieu sur des entre-nœuds âgés de 7 à 11 ans.

Nombre de niveaux d'ottoques
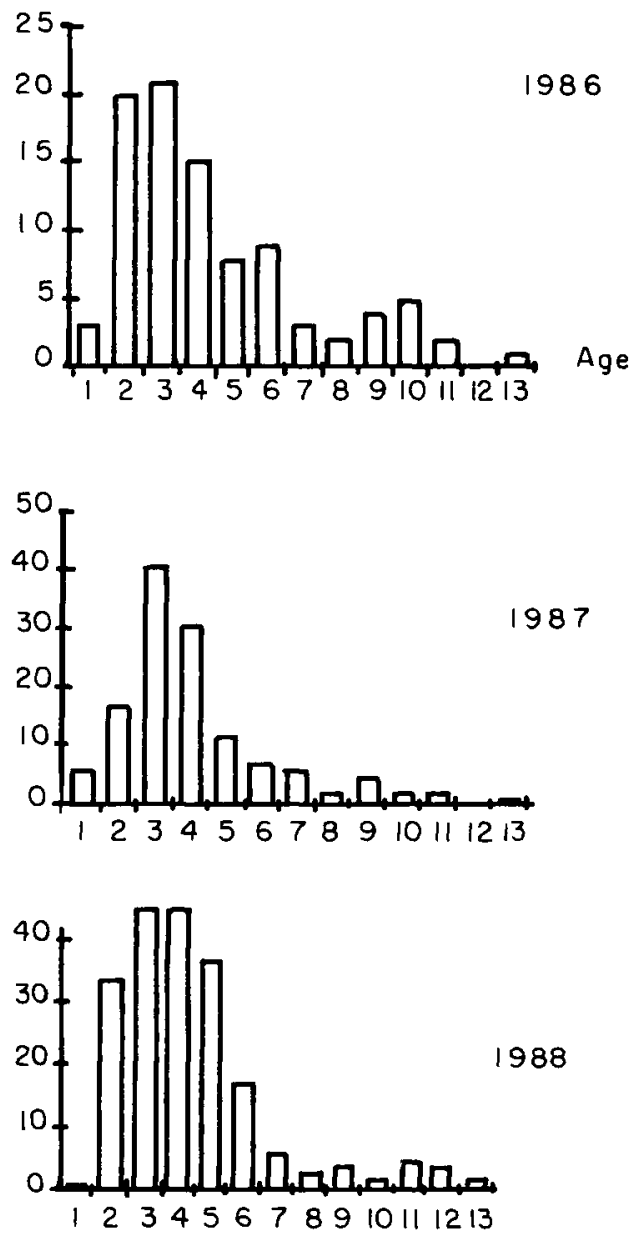

Fig 1. Nombre d'attaques de Dioryctria sylvestrella en fonction de l'âge de la zone de l'arbre infestée. 
Dans 29 et $50 \%$ des cas, respectivement sur les parties de l'arbre âgées de 2 à 6 ans et de 7 à 13 ans, les attaques sont situées au niveau de l'entre-nœud (contre 71 et $50 \%$ sur verticille). Le test du $\chi^{2}$ avec correction pour la continuité permet de préciser que les différences entre ces pourcentages sont significatives au seuil de probabilité de $5 \%$. Ainsi, sur les pousses âgées de plus de 7 ans, les attaques se situent indifféremment au niveau du verticille ou sur l'entre-nœud. La zone de plus grande sensibilité semble donc correspondre aux verticilles âgés de moins d'une dizaine d'années.

Ces observations confirment celles faites antérieurement (Suéron, 1982).

\section{Relations entre critères dendrométriques et niveau d'infestation}

\section{Évolution générale des taux d'attaque}

D'une parcelle à l'autre, les taux d'attaque sont très variables. L'évolution pluriannuelle présente également une très grande variabilité selon les peuplements. La figure 2 rend compte de cette évolution dans chaque parcelle.

\section{Discrimination des peuplements par I'AFD}

Cette analyse, effectuée sur la totalité des arbres étudiés ou l'ensemble des arbres attaqués, aboutit au même résultat.

La figure 3 représente la position des placettes dans le plan des 2 axes canoniques. L'axe canonique 1 représente 93\% de la discrimination entre peuplements et est corrélé essentiellement à l'accroissement en surface terrière $\Delta g$, mais aussi au taux d'attaque $\mathrm{T}_{\mathrm{a}}$ (le coefficient de corrélation inter-placettes entre variable canonique et $\Delta g$ vaut 0,99 et, entre variable cano-

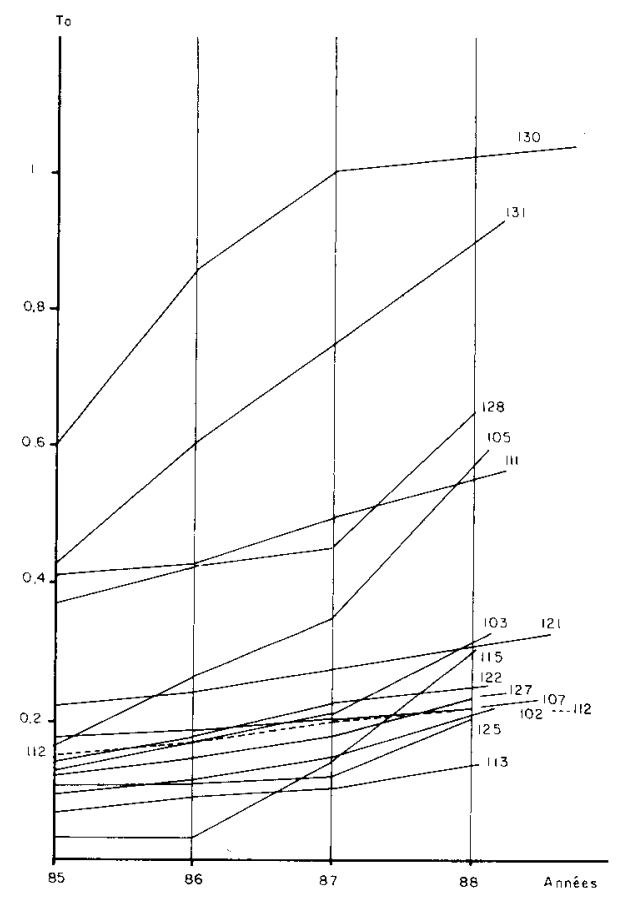

Fig 2. Évolution du nombre d'attaques Ta par arbre entre 1985 et 1988 sur les 15 placettes.

nique et $\left.T_{a}, 0,62\right)$. L'axe 2 discrimine les placettes sur leur taux d'attaque (le coefficient de corrélation inter-placettes entre variable canonique et $T_{a}$ vaut 0,78 ).

\section{Relations entre type de station (lande), productivité et niveau d'infestation}

Les moyennes du taux d'attaque $T_{a}$ pour la période 1980-1988 des peuplements de même type de lande $(0,5,0,4,0,3$ respectivement pour $L H, L M S$ et LS) ne sont pas significativement différentes au seuil de $5 \%$.

Il paraît donc difficile de considérer les conditions stationnelles ainsi définies comme un bon prédicteur du taux d'attaque. 


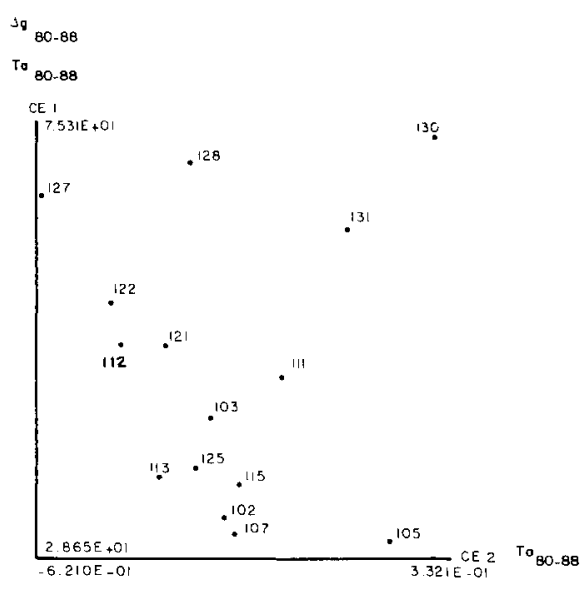

Fig 3. Analyse factorielle discriminante sur la totalité des arbres des 15 peuplements, avec les 2 variables : accroissement en surface terrière $\Delta \mathrm{g}_{80-88}$ et taux d'attaque par arbre $\mathrm{Ta}_{80-88}$.

\section{Relations entre taux d'attaque et caractéristiques de peuplement}

Pour chacune des périodes, 1980-1985 et 1980-1988, une régression multiple descendante a été effectuée (tableau III), mettant en jeu :

-1 variable expliquée: $T_{\mathrm{a}}$;

-5 variables explicatives: $\mathrm{H}_{16}, \overline{\Delta \mathrm{g}}, \Delta \mathrm{h}_{\text {dom }}$, $N_{\text {moy }}, L_{\text {moy. }}$

Le coefficient de corrélation multiple est significatif au seuil de $5 \%$ à partir du $4^{e}$ palier (test F) qui comprend 2 variables explicatives: l'indice de vigueur $\mathrm{H} 16$ et l'accroissement en surface terrière $\overline{\Delta g}$. L'apport de H16 n'est pas significatif. Ainsi, pour les périodes $1980-1985$ et 19801988 , respectivement $62 \%$ et $58 \%$ de la variabilité de $T_{a}$ est expliquée par $\overline{\Delta g}$ (fig 4).

Tableau III. Résultats de l'analyse de variance de la régression multiple descendante (équation [1]).

\begin{tabular}{|c|c|c|c|c|}
\hline $\begin{array}{l}\text { Rég Mul Des * } \\
\text { Période 80-85 }\end{array}$ & $\begin{array}{c}\text { Degrés } \\
\text { de liberté }\end{array}$ & $\begin{array}{l}\text { Carrés } \\
\text { Moyens }\end{array}$ & Tests $F$ & $R^{2}$ \\
\hline $\begin{array}{l}\text { Palier } 1 \\
\mathrm{~N}_{\text {moy }}, \mathrm{L}_{\text {moy }}, \\
\mathrm{H}_{16}, \overline{\Delta \mathrm{g}}, \Delta \mathrm{h}_{\text {dom }}\end{array}$ & 5 & 0,042 & 2,39 (NS) & 0,75 \\
\hline $\begin{array}{l}\text { Palier } 2 \\
\text { var élim } \\
N_{\text {moy }}\end{array}$ & $\begin{array}{l}4 \\
1\end{array}$ & $\begin{array}{l}0,052 \\
0,006\end{array}$ & $\begin{array}{c}2,90 \\
0,32 \text { (NS) }\end{array}$ & 0,74 \\
\hline $\begin{array}{l}\text { Palier } 3 \\
\text { var élim } \\
L_{\text {moy }}\end{array}$ & $\begin{array}{l}3 \\
1\end{array}$ & $\begin{array}{l}0,067 \\
0,007\end{array}$ & $\begin{array}{c}3,74 \\
0,39 \text { (NS) }\end{array}$ & 0,73 \\
\hline $\begin{array}{l}\text { Palier } 4 \\
\text { var élim } \\
\Delta h_{\text {dom }}\end{array}$ & $\begin{array}{l}2 \\
1\end{array}$ & $\begin{array}{l}0,084 \\
0,031\end{array}$ & $\begin{array}{c}4,73^{\star} \\
1,76 \text { (NS) }\end{array}$ & 0,67 \\
\hline $\begin{array}{l}\text { Palier } 5 \\
\text { var élim } \\
\mathrm{H}_{16}\end{array}$ & $\begin{array}{l}1 \\
1\end{array}$ & $\begin{array}{l}0,143 \\
0,025\end{array}$ & $\begin{array}{c}8,04^{*} \\
1,42 \text { (NS) }\end{array}$ & 0,62 \\
\hline Écart à la régression & 9 & 0,018 & & \\
\hline
\end{tabular}

\footnotetext{
* Règ Mul Des: régression multiple descendante.
} 


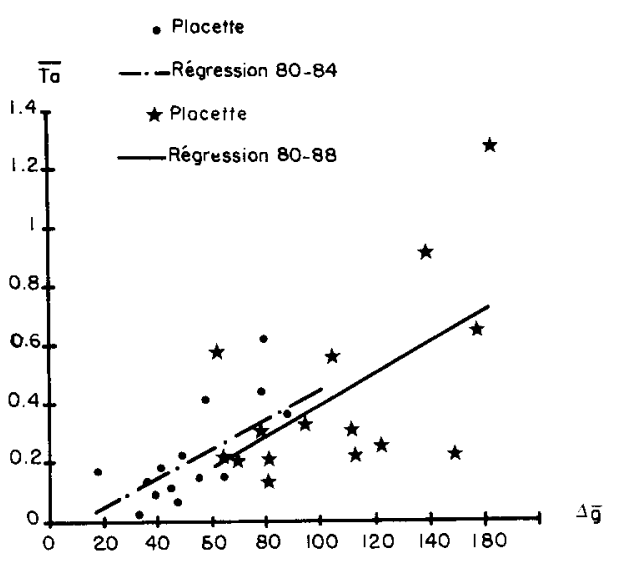

Fig 4. Relation entre le nombre moyen d'attaques $T_{a}$ par arbre et l'accroissement moyen en surface terrière $\overline{\Delta g}$ (équations [1] et [2]).

Équation de la droite de régression (période 1980 à 1985) :

$$
\mathrm{T}_{\mathrm{a}}=-0,039+0,00481 * \overline{\Delta \mathrm{g} 80-84}
$$

Équation de la droite de régression (période 1980 à 1988) :

$$
\mathrm{T}_{\mathrm{a}}=-0,135+0,00499 * \overline{\mathrm{gg}} 80-88
$$

Les caractéristiques du peuplement autres que $\overline{\Delta g}$ ne s'avèrent pas de bons prédicteurs du taux d'attaque $T_{\mathrm{a}}$. L'âge de référence choisi ici (16 ans) pour l'indice de vigueur est sans doute trop bas, mais les peuplements étant encore jeunes, l'estimation de la hauteur dominante à 40 ans n'a pu être effectuée qu'avec une erreur d'extrapolation notable. L'indice de concurrence ou la moyenne des densités sur une période donnée ne présentent pratiquement pas de variabilité. Les normes sylvicoles concernant les éclaircies sont généralement respectées, de sorte que les peuplements se caractérisent à ces âges par une croissance proche de la croissance libre. L'accroissement en surface terrière exprime seulement $60 \%$ de la variabilité du taux d'attaque au niveau du peuplement et ne peut être retenu comme prédicteur. En revanche, il traduit bien un phénomène au même titre que la relation que nous avons trouvée au niveau individuel: le coefficient de régression intra-peuplement pour la période 19801988 calculé à partir de la matrice des carrés et coproduits moyens intra, équivaut à 0,0045 , valeur proche de celles trouvées au niveau peuplement (équations [1] et [2]).

Les peuplements ayant un fort taux de croissance absolu sont plus attaqués que les autres et au sein d'un peuplement, les arbres ayant un forte croissance cambiale sont les plus attaqués.

\section{DISCUSSION ET CONCLUSION}

Ce travail a permis d'aborder différents problèmes méthodologiques concernant l'évaluation du taux d'attaque et la détermination de facteurs discriminants. Les 15 peuplements échantillonnés initialement pour leurs caractéristiques de station et leur vigueur offrent une bonne représentativité de la productivité et du taux d'infestation dans la région de Pissos. Les peuplements de plus forte croissance sont les plus fortement infestés, les arbres aussi. Les mesures dendrométriques et les notations faites sur ces jeunes peuplements sont encore trop récentes pour qu'il soit possible de présumer du type de liaison entre productivité et dynamique de population de Dioryctria sylvestrella dans ces peuplements. II faut cependant souligner le fait que les attaques actuelles se situent dans les 2 tiers supérieurs de la partie commercialisable du fût.

II importe désormais de définir un seuil économique de nuisibilité. L'impact sur la qualité du bois d'une cicatrisation après une attaque se traduisant par la présence 
de nodules de résine et des incorporations de rhytidome doit être étudié.

La pratique de l'élagage, qui tend à se généraliser en sylviculture intensive, risque de favoriser l'installation des chenilles de la pyrale sur les plaies consécutives à cette technique. II est donc essentiel désormais de définir un programme d'étude afin de déterminer les périodes optimales d'élagage.

Les 2 principaux résultats de cette étude, à savoir que les attaques sont localisées essentiellement au niveau de pousses jeunes (2 à 6 ans) et sur des arbres de forte croissance cambiale, sont complémentaires et permettent d'envisager des relations spécifiques tissu-insecte. Nous pouvons notamment formuler plusieurs hypothèses sur les caractéristiques anatomiques et structurales de l'écorce de différentes parties du fût, en relation avec certains stades du cycle biologique de l'insecte.

La ponte, puis la pénétration de la larve dans le liber pourraient être facilitées par l'existence :

- d'une frange de liber mise à nu à l'intérieur des nombreuses anfractuosités du tronc, notamment au niveau de l'insertion des branches ;

- d'un rhytidome fin ; celui-ci existe sur les zones encore jeunes (pousses de 2 à 6 ans). Une étude sur les caractéristiques anatomiques de l'écorce de l'épicéa commun a mis en évidence une diminution de l'épaisseur du rhytidome avec la hauteur, c'est-à-dire avec l'âge des tissus (Nihoul et al, 1989). Sur pin maritime, ce phénomène peut être semblable. Le rhytidome fin existe aussi sur des bourrelets de cicatrisation, par exemple au niveau de plaies de gélivure, zones sur lesquelles de nombreuses attaques ont été observées (36\% des arbres gélivés, contre seulement $0,5 \%$ des arbres indemnes ont été attaqués).

Le développement et la croissance de la larve pourraient dépendre de la quantité et de la qualité du liber. L'influence majeure de l'épaisseur et de la qualité nutritive du phloème sur la reproduction d'ips calligraphus a déjà été montrée (Haack et al, 1987 ; Poop et al, 1989). Nihoul et al (1989) ont par ailleurs également constaté une augmentation relative des tissus vivants du liber avec la position en hauteur.

Des études anatomiques et structurales de l'écorce doivent être envisagées et être intégrées au programme de sélection de pin maritime en distinguant le niveau génétique individuel intra- et inter-provenance. Des observations ont déjà montré que le pin maritime corse de forte productivité était 4 à 5 fois plus attaqué que le pin maritime landais. Des éclaircissements sur l'origine des différences génétiques enregistrées permettraient peut-être de disposer de prédicteurs de moindre sensibilité à Dioryctria sylvestrella.

L'influence des parasites et des prédateurs sur ce ravageur est encore trop peu connue. Les efforts sur l'étude de la biologie et la maîtrise de l'élevage de la pyrale du tronc doivent avant tout être poursuivis. Une meilleure compréhension des relations plante-insecte en dépend.

\section{RÉFÉRENCES}

Baradat $\mathrm{P}$ (1989) Amélioration génétique des arbres forestiers. Élements méthodologiques. INRA, Recherches forestières, laboratoire amélioration, Pierroton, $204 p$

Baradat P, Pastuzka P (1990) Stratégies d'amélioration et diversification variétale du pin maritime. Colloque Arbora, Bordeaux, tome 2 , 375-390

Haack RA, Wilkinson RC, Foltz JL, Corneil JA (1987) Spatial attack pattern, reproduction, and brood development of Ips calligraphus (Coleoptera: Scolytidae) in relation to Slash pine thickness: a field study. Environ Entomol 16, 428-436

IFN (1971) Département de la Gironde, 1961 
IFN (1992) Département de la Gironde, résultats du $3^{3}$ inventaire forestier, 1987

Lemoine B (1991) Growth and yield of maritime pine (Pinus pinaster, Ait): the average dominant tree of the stand. Ann Sci For 48, 593-611

Ménassieu P, Stockel J, Levieux J (1989) Données actuelles sur la biologie de Dioryctria sylvestrella (Ratz) (Lepidoptera: Pyralidae), ravageur du pin maritime Pinus pinaster (Ait) dans le sud-ouest de la France. J Appl Entomol 107 (3): 238-247

Nihoul P, Nef J, Waterkeyn L (1989) Variabilité inter- et intra- individuelle de quelques caractéristiques anatomiques de l'écorce de l'épi- céa commun (Picea abies ( $L$ ) Karsten), en Ardenne belge. Ann Sci For 46 (1), 85-95

Popp MP, Wilkinson RC, Jokela EJ, Harding RB, Phillips TW (1989) Effects of Slash pine phloem nutrition on the reproductive performance of Ips calligraphus (Coleoptera Scolytidae). Environ Entomol 18 (5), 795-799

Saint-Didier J (1976) Nature et évolution de la végétation de la Grande Lande à Pissos. Mémoire ENITA Dijon, $110 p$

Suéron C (1982) Aspects génétiques et biochimiques de la résistance de Pinus pinaster (Ait) à Dioryctria sylvestrella (Ratz). Mémoire ENITEF, $126 p$ 